\title{
Research Paper: Effects of Transcutaneous Electrical Nerve Stimulation on Voluntary Quadriceps Activation in Healthy Amateur Female Athletes
}

\author{
Horieh Sadeghi' ${ }^{1}$, Seyed Mohsen Mir $^{1^{*}}$ Q, Kazem Malmir ${ }^{1}$ @ , Nastaran Ghotbi ${ }^{1}$
}

1. Department of Physiotherapy, School of Rehabilitation, Tehran University of Medical Sciences, Tehran, Iran.

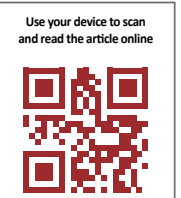

citation: Sadeghi H, Mir SM, Malmir K, Ghotbi N. Effects of Transcutaneous Electrical Nerve Stimulation on Voluntary Quadriceps Activation in Healthy Amateur Female Athletes. Journal of Modern Rehabilitation. 2019; 13(4):215-220. http:// dx.doi.org/10.32598/JMR.13.4.215

http://dx.doi.org/10.32598/JMR.13.4.215

Article info:

Received: 05 May 2019

Accepted: 27 Jul 2019

Available Online: 01 Oct 2019

Keywords:

Electrical nerve stimulation (TENS), Quadriceps, Voluntary muscle activation, Amateur athlete

\section{ABSTRACT}

Introduction: Compared with professionals and semi-professionals, amateur athletes need more strengthening modalities due to their lower muscle strength, as well as limited facilities and time. Transcutaneous Electrical Nerve Stimulation (TENS) is a sensory-targeted modality traditionally used to treat pain, but it is also one of the most successful methods for increasing muscle activation level, too.

Materials and Methods: A total of 30 sportswomen in basketball, volleyball, futsal, football, and taekwondo with the Mean \pm SD age of $23.5 \pm 3.6$ years were randomly divided into two groups: intervention $(\mathrm{n}=15)$ and control $(\mathrm{n}=15)$. In the intervention group, a single session of TENS with a frequency of $150 \mathrm{~Hz}$ and pulse duration of $150 \mu$ s was applied for 20 minutes. The control group received placebo TENS. Isokinetic dynamometer and vertical jump test were used to assess quadriceps activation and strength. All samples were evaluated at three different time intervals: before, immediately after, and 30 minutes after the intervention.

Results: The mean maximal voluntary isometric contraction, central activation ratio, and height of jumping in the TENS and control group were not significantly different immediately and 30 minutes after the intervention $(\mathrm{P}>0.05)$.

Conclusion: According to this study, a single session of TENS cannot immediately improve quadriceps activation and strength in amateur female athletes. 


\section{Introduction}

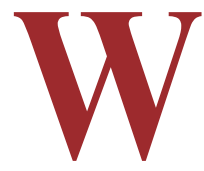

ith rising people's interests in sports around the world, sports injuries are also increasing. Knee injuries account for one-quarter of all sports injuries and take the longest time and efforts to heal [1].

The chance of injuries in female athletes is higher for several reasons; for example, females demonstrate greater quadriceps activation compared with males during hopping, cutting, and lunging maneuvers. Also, females generally have smaller and less developed muscles as joint stabilizers. Finally, females have weaker hamstring muscles relative to their quadriceps, and this imbalance increases the risk of ligaments injuries [2].

Among the intrinsic injury risk factors including age, sex, phase of the menstrual cycle, ligamentous laxity, muscle strength, flexibility, and the like, "muscle strength" is a variable that not only reduces the chance of injury but also plays a role in improving the performance of the athlete [3]. Because quadriceps activity is high in most sports' tasks such as jumping, sprinting, squat, and son on, physical therapists and sports scientists can focus on increasing the quadriceps strength to prevent injury and even to improve athletic performance.

Furthermore, amateurs or semi-professional athletes generally have lower strength levels compared with professional athletes, and thus will gain more benefits from an effective strength training program. Professional athletes have the advantage of incorporating different training protocols, but amateur athletes lack this opportunity because of their responsibilities such as school, occupation, and family. So a short and effective program that can enhance lower body strength and performance would be of great benefit to amateur team sport athletes [4].

Various training modalities can be used for improving muscular strength. In particular, electromyostimulation (EMS) is employed as a means of strength training in healthy humans. An increase in muscle strength was evident in many muscle groups after EMS training ranging from $10 \%$ to $41 \%$, including quadriceps muscles. Moreover, EMS training improves specific soccer tasks. Thus, besides traditional soccer training, an EMS training program for 3 to 5 weeks would be a viable means for improving force and specific soccer tasks before and during the play season $[5,6]$. It is hypothesized that focal knee joint cooling may facilitate the quadriceps motoneuron pool by increasing excitatory afferent stimuli [7].
Transcutaneous Electrical Nerve Stimulation (TENS) is a sensory-targeted modality traditionally used to treat pain, but it is also one of the most successful methods for increasing quadriceps activation levels after a single session in patients with quadriceps activation failure [810]. TENS can reduce arthrogenic muscle inhibition by postsynaptic inhibition of the Ib inhibitory in knee osteoarthritis, meniscus tearing, and so on [11]. Although increasing in quadriceps motoneuron pool excitability using the Hoffmann reflex or Central Activation Ratio (CAR) have been reported following the TENS application, but no study has examined it in healthy athletes.

An intervention that is successful in disinhibiting the motoneuron pool, allowing for increased muscle activation in injured athletes, may be useful for facilitating and thus leading to more optimal strength and function in uninjured athletes. So this study was designed to change Voluntary Quadriceps Activation (VQA) and quadriceps strength by a single session in healthy amateur female athletes. We hypothesized that quadriceps CAR and strength would increase after 20 minutes of TENS compared with a control condition.

\section{Methods}

A crossover, repeated-measures design was used in this study. The independent variables were treatment and time. Treatment had two levels (TENS and control group) and 3 different time intervals (before the intervention, immediately after the intervention, and $30 \mathrm{~min}-$ utes after the intervention).

The primary outcome measurement was voluntary quadriceps activation expressed as Central Activation Ratio (CAR); secondary outcome measurements were quadriceps knee extension torque or Maximal Voluntary Isometric Contraction (MVIC) and vertical jump test. All athletes were blinded to study.

\section{Study subjects}

A total of 30 healthy amateur female athletes in basketball, volleyball, futsal, football, and taekwondo, who studied at the faculty of physical education, volunteered for this study.

None of the participants had a history of lumbar or lower extremities operation, deformity, and injury (during the last 6 months). The participants did not use corticosteroid or hyaluronic acid injection, neurological, and analgesics drugs during the previous 24 hours. Subjects would also be excluded if they felt pain in the trunk or 
lower extremities; were dissatisfied with study; did not complete the tests or did not tolerate TENS and exogenous electrical stimulation.

\section{Study procedure}

All of the tests and interventions were performed in a single session. Subjects warmed up on a stationary bicycle at a self-selected speed for 5 minutes. The dominant leg, determined by asking the participant with which leg they preferred to kick a ball, was tested in all participants.

Maximal voluntary isometric contraction and central activation ratio

We used a Biodex System 3 (Biodex Medical System INC., Shirley, New York, USA) dynamometer to calculate the central activation ratio (CAR). Subjects sat on an isokinetic dynamometer with $85^{\circ} \mathrm{C}$ hip flexion and $60^{\circ} \mathrm{C}$ knee flexion. Their shoulders and pelvis belts were closed to prevent any additional movements. The mechanical axis of the dynamometer is aligned with the anatomical axis of the knee [12]. Two $4.5 \times 6 \mathrm{~cm}$ carbon electrodes were used to deliver the stimulus to the femoral nerve. The anode was positioned at the height of the greater femoral trochanter, and the medial electrode border was in line with the anterosuperior iliac spine. The cathode was positioned $3 \mathrm{~cm}$ above the patella [13].

Three trials of knee isometric extension with maximal effort were performed, and participants asked to hold the contraction for $7 \mathrm{~s}$ with a 60 -s rest. Then maximum torque and force were recorded (FMVIC). All MVIC values were reported as $\mathrm{Nm} / \mathrm{kg}$. All participants were verbally encouraged to use their power during knee extension. Before starting the test, the participants performed 3 submaximal knee extension to be familiarized with the test [14]. Equation 1 shows how FMVIC is computed:

\section{Tq MVIC - Momentum leg - Momentum foot Radian $60^{\circ} \times$ Lever arm Quadriceps}

Novin (735x) stimulator produced the exogenous electrical stimulus. A stimulus with a frequency of $50 \mathrm{~Hz}$, pulse duration of $300 \mu \mathrm{s}$ and the intensity of muscle contraction was delivered to the participant's leg when quadriceps contraction reaches maximum force plateau to stimulate a more strong muscle contraction. Three trials of knee extension with maximal effort were performed, and the participant was asked to hold it for $9 \mathrm{~s}$ with a 60 -s rest. The recorded force, named "superimposed burst force" or "FSIB."

\section{Tq MVIC - Momentum leg - Momentum foot Radian $60^{\circ} \times$ Lever arm Quadriceps}

The central activation ratio was calculated by dividing the Force Measurements of the Maximal Voluntary Contraction (FMVIC) by that of the Force produced by the Superimposed Burst (FSIB) plus the maximal voluntary contraction (FMVIC) [15]:

$$
C A R=\frac{F M V I C}{F M V I C+F S I B}
$$

CAR is used to assess voluntary activation of a muscle by measuring the recruitment of motor units and frequency of motor unit firing.

\section{Vertical jump}

A vertical jump was used to assess the explosive strength of the lower limbs following a stretch of the agonist muscles [16]. The subject would stand next to a smooth, dark wall on the floor and was asked to stretch out her upper limb and reach as high as possible with either hand and makes a mark on the wall with chalk (H1). Holding the desired jump position with the preferred side to the wall, the subject jumped as high as possible and made another mark at the peak of the jump (H2). The vertical jump score was calculated based on the difference between the two marks. Three trials of the test were performed with a rest period of 2 minutes, and the maximum height was recorded [17].

\section{Study intervention}

Subjects were randomly assigned into two groups: intervention $(\mathrm{n}=15)$ and control $(\mathrm{n}=15)$ after baseline tests. The TENS unit (Arman Pooya, model FA720) was used in both the intervention and control groups. Active TENS consisted of a sensory continuous, biphasic current (frequency of $150 \mathrm{~Hz}$, and pulse duration of $150 \mu \mathrm{s})$ [15]. Four separate $4.5 \times 6 \mathrm{~cm}$ electrodes were used on the medial and lateral superior as well as medial and lateral inferior borders of the patella to deliver the TENS stimulation to knee joint of the participants for 20 minutes. TENS intensity was increased to the highest tolerable sensory stimulation without causing muscle contraction. Placebo TENS was administered with the same instruments, but after 30 seconds, the current discontinued. All other features of the placebo unit were identical [14]. 
The participants of the two groups were immediately evaluated after the intervention with the same tests as before. The results were recorded for the calculations. Then participants rested and assessed for the third time 30 minutes after the end of the intervention.

\section{Statistical analysis}

Three separate Analyses of Variance (ANOVA) of the group (TENS and placebo TENS) by time (before the intervention, immediately after the intervention, and 30 minutes after the intervention) were used to evaluate differences in CAR, MVIC and Vertical Jump Test (VJT). Means and standard deviation were calculated for all outcome measures before data analysis. P values less than 0.05 were considered significant in all experiments.

\section{Results}

Thirty athletes were volunteered to participate in this study (Mean \pm SD age: $23.53 \pm 0.36 \mathrm{y}, \mathrm{Mean} \pm$ SD height: $167 \pm 3.4 \mathrm{~cm}, M e a n \pm$ SD weight: $60.02 \pm 0.3 \mathrm{~kg}$ ). The dominant leg of all participants was the right leg.

No significant differences were found between groups in any of the participants' demographics $(\mathrm{P}>0.05)$.

There were no statistical differences in the baseline, immediately after and 30 minutes after the intervention in $\operatorname{MVIC}(\mathrm{P}=0.85, \mathrm{~F}=0.091)$ and $\mathrm{CAR}(\mathrm{P}=0.190, \mathrm{~F}=1.766)$ and vertical jump $(\mathrm{P}=0.153, \mathrm{~F}=2.076)$.

\section{Discussion}

This study aimed to investigate the effect of TENS on quadriceps activation in healthy amateur female athletes. The results showed that a single application of TENS does not improve the strength and quadriceps activation of amateur athletes, and does not affect the sport tasks such as vertical jump.

TENS is an inexpensive, non-pharmaceutical, non-invasive, and simple intervention. The parameters of TENS that are used to improve muscle activation are identical with the parameters used to reduce pain, which indicates that modality that was used to relieve pain in the past could have a new and independent application. TENS can minimize muscle inhibition via postsynaptic inhibition of the Ib interneuron; in other words, by reducing the activity of the inhibitory interneurons of the motoneuron pool, it reduces arthrogenic muscle inhibition $[18,19]$.
Applying high-frequency TENS after open meniscectomy and Anterior Cruciate Ligament (ACL) reconstruction increases quadriceps activation during subsequent maximal voluntary contractions. Furthermore, Hopkins and coworkers (2002) have found that high-frequency TENS $(120 \mathrm{~Hz}$, pulse duration of $0.1 \mathrm{~s})$ prevents the decline in quadriceps H-reflex amplitude seen after the infusion of fluid into the knee joint. Recently, the application of high-frequency $(150 \mathrm{~Hz}$, pulse duration of 0.15 s) TENS to osteoarthritis knee joints has been shown to significantly improve quadriceps activation. Low-frequency (4 Hz, pulse duration of $1 \mathrm{~s}$ ), acupuncture-like TENS, has been reported to increase quadriceps force output by $71 \%$ in osteoarthritis patients, but it has remained unknown whether low-frequency TENS may be effective in reducing Arthrogenic Muscle Inhibition (AMI) in patients with other knee joint pathologies [20].

In this study, the isokinetic dynamometer was used to measure quadriceps torque that showed no significant effect of TENS on the improvement of MVIC. Vanderthommen et al. used Neuromuscular Electrostimulation (NMES) with a frequency of $80 \mathrm{~Hz}$ and a pulse duration of $0.35 \mathrm{~ms}$ for a single session of 20 minutes to change quadriceps and hamstring torque. It was not only effective but also reduced muscle torque and impaired muscle function [21]. Dehail and colleagues reviewed articles to identify the effect of the application of EMS on strength gain and suggested superimposed or combined EMS techniques are generally more efficient than EMS alone [22]. In another study, concurrent use of EMS and plyometric exercise increased quadriceps strength, the amount of the jump, and speed of a sprint, but a single application of EMS decreased speed of sprint [23]. The only study used TENS to increase the strength of the quadriceps muscle in healthy people is Onigbinde et al. study. They used TENS current at $80 \mathrm{~Hz}$ and pulse duration of $100 \mu$ s for 8 weeks, twice per week, and 15 minutes per day. The result was an increase in ipsilateral and contralateral muscle strength [24].

There are several methods of muscle activation assessment, such as EMG, SIB technique, and Interpolated Twitch Technique (ITT). In this study, the SIB technique showed that TENS did not improve quadriceps activation. Glaviano and colleagues investigated the effect of using Percutaneous Electrical Nerve Stimulation (PENS) with a frequency of $50 \mathrm{~Hz}$ and a pulse duration of $70 \mu \mathrm{s}$ on quadriceps and hamstring strength in injured knees. Measurement of strength and muscle activation by isokinetic indices showed that PENS could not increase muscle torque and activation compared with the control group [25]. In another study, a single session of PENS in 
the gluteus medius and vastus medialis improved functional tasks such as step down and single-leg squat in EMG monitoring [26].

Among several functional quadriceps strength tests, the vertical jump is a valid test for evaluating quadriceps strength and performance, especially in soccer and basketball players [27]. In this study, TENS did not improve the height of the vertical jump test. Stania et al. used a single session of NMES and whole-body vibration (both with a frequency of $40 \mathrm{~Hz}$ ) on quadriceps and suggested that electrical or mechanical stimulation cannot improve the height of vertical jump [28]. Pillard et al. stimulated quadriceps with NMES for 1 week and then observed the increase in the height of jump [29].

We hypothesized that TENS with a similar disinhibitory mechanism of injuries could facilitate quadriceps by increasing presynaptic excitatory potentials and motor output. Also, the mechanism of focal knee joint cooling in increasing quadriceps activation in healthy subjects was the same.

One of the reasons for our negative result may be the single session of stimulation, and if several sessions of stimulation were used (with cumulative effect), different results might be expected. Although the SIB technique is sensitive to changes in muscle activation after interventions or treatments, valid CAR estimates rely on the ability to effectively isolate knee extensors to prevent contributing muscles other than the quadriceps generating maximal torque during a voluntary effort. Suboptimal electric stimulation settings may also cause invalid estimates of quadriceps activation if the stimulation fails to fully activate all motor units in a motoneuron pool [30]. So if another measurement tool was used, such as EMG, the results might have been different. Besides, in this study, criteria for choosing amateur athletes were based on their words, and the lack of awareness of the participants' sports activities level can affect the results.

\section{Conclusion:}

A single session of TENS could not improve quadriceps strength, activation, and performance in healthy amateur female athletes.

This study was single-blinded so that results may be biased. Although participants were selected from different sports and no comparison was made between two genders.
Given the effect of cryotherapy on quadriceps strength of healthy athletes, a study could be designed to investigate the impact of concurrent of TENS and cryotherapy. Also, further research is recommended to determine the effect of TENS after more sessions with a cumulative effect.

\section{Ethical Considerations}

Compliance with ethical guidelines

The Ethics Committee of Tehran University of Medical Sciences approved the study (Code: IR.TUMS.FNM. REC.1398.048). Subjects signed informed consent forms before participating in the study.

Funding

The present paper was extracted from the MSc thesis of the first author, Department of Physiotherapy, School of Rehabilitation, Tehran University of Medical Sciences.

\section{Authors contributions}

All authors contributed in preparing this paper.

\section{Conflict of interest}

The authors declared no conflict of interest.

\section{Acknowledgements}

The authors would like to acknowledge their gratitude to the Department of Physiotherapy at Tehran University of Medical Sciences.

\section{References}

[1] Spanier R. The evolution and prevalence of knee injuries: Repair at what cost? 2014;5(1):120-30. [DOI:10.18357/ar.spanierr.512014]

[2] Deda N, Kalaja A. Epidemiology in knee injuries in volleyball players. European Scientific Journal. 2015; 11(15):44-52. https:/ / eujournal.org/index.php/esj/article/view/5614/5405

[3] Dugan SA. Sports-related knee injuries in female athletes: What gives? American Journal of Physical Medicine \& Rehabilitation 2005; 84(2):122-30. [DOI:10.1097/01.PHM.0000154183.40640.93] [PMID]

[4] Lockie RG. A 6-week base strength training program for sprint acceleration development and foundation for future progression in amateur athletes. Strength \& Conditioning Journal. 2018; 40(1):2-12. [DOI:10.1519/SSC.0000000000000341]

[5] Maffiuletti NA, Cometti G, Amiridis IG, Martin A, Pousson $\mathrm{M}$, Chatard JC. The effects of electromyostimulation training and basketball practice on muscle strength and jumping abil- 
ity. International Journal of Sports Medicine. 2000; 21(6):437-43. [DOI:10.1055/s-2000-3837] [PMID]

[6] Seyri K, Maffiuletti NA. Effect of electromyostimulation training on muscle strength and sports performance. Strength and Conditioning Journal. 2011; 33(1):70-5. [DOI:10.1519/ SSC.0b013e3182079f11]

[7] Pietrosimone BG , Ingersoll CD. Focal knee joint cooling increases the quadriceps central activation ratio. Journal of Sports Sciences. 2009; 27(8):873-9. [DOI:10.1080/02640410902929374] [PMID]

[8] Son SJ. Effects of transcutaneous electrical nerve stimulation on voluntary quadriceps activation and vertical ground reaction force during walking in subjects with experimental knee pain [MSc. thesis]. Provo, UT: Brigham Young University; 2014. https://scholarsarchive.byu.edu/cgi/viewcontent. cgi?article $=6273 \&$ context $=$ etd

[9] Hopkins J, Ingersoll CD, Edwards J, Klootwyk TE. Cryotherapy and transcutaneous electric neuromuscular stimulation decrease arthrogenic muscle inhibition of the vastus medialis after knee joint effusion. Journal of Athletic Training. 2002; 37(1):25-31. [PMID] [PMCID]

[10] Pietrosimone BG, Hart JM, Saliba SA, Hertel J, Ingersoll CD. Immediate effects of transcutaneous electrical nerve stimulation and focal knee joint cooling on quadriceps activation. Medicine \& Science in Sports \& Exercise. 2009; 41(6):1175-81. [DOI:10.1249/ MSS.0b013e3181982557] [PMID]

[11] Hopkins J, Ingersoll CD, Edwards J, Klootwyk TE. Cryotherapy and transcutaneous electric neuromuscular stimulation decrease arthrogenic muscle inhibition of the vastus medialis after knee joint effusion. Journal of Athletic Training. 2002; 37(1):25-31. [PMID] [PMCID]

[12] Pietrosimone BG, Saliba SA. Changes in voluntary quadriceps activation predict changes in quadriceps strength after therapeutic exercise in patients with knee osteoarthritis. The Knee. 2012; 19(6):939-43. [DOI:10.1016/j.knee.2012.03.002] [PMID]

[13] Pietrosimone BG, Selkow NM, Ingersoll CD, Hart JM, Saliba SA. Electrode type and placement configuration for quadriceps activation evaluation. Journal of Athletic Training. 2011; 46(6):621-8. [DOI:10.4085/1062-6050-46.6.621] [PMID] [PMCID]

[14] Pietrosimone BG, Saliba SA, Hart JM, Hertel J, Kerrigan DC, Ingersoll $\mathrm{CD}$. Effects of transcutaneous electrical nerve stimulation and therapeutic exercise on quadriceps activation in people with tibiofemoral osteoarthritis. Journal of Orthopaedic \& Sports Physical Therapy. 2011; 41(1):4-12. [DOI:10.2519/jospt.2011.3447] [PMID]

[15] Pietrosimone BG, Saliba SA, Hart JM, Hertel J, Ingersoll CD Contralateral effects of disinhibitory tens on quadriceps function in people with knee osteoarthritis following unilateral treatment. North American Journal of Sports Physical Therapy. 2010; 5(3):111-21. [PMID] [PMCID]

[16] Delecluse C, Roelants M, Diels R, Koninckx E, Verschueren S. Effects of whole body vibration training on muscle strength and sprint performance in sprint-trained athletes. International Journal of Sports Medicine. 2005; 26(8):662-8. [DOI:10.1055/s-2004-830381] [PMID]

[17] Klavora P. Vertical-jump tests: A critical review. Strength and Conditioning Journal. 2000; 22(5):70-4. [DOI:10.1519/00126548200010000-00020]
[18] Vance CGT, Dailey DL, Rakel BA, Sluka KA. Using TENS for pain control: The state of the evidence. Pain Management. 2014; 4(3):197-209. [DOI:10.2217/pmt.14.13] [PMID] [PMCID]

[19] DeSantana JM, Walsh DM, Vance C, Rakel BA, RN, Sluka KA. Effectiveness of transcutaneous electrical nerve stimulation for treatment of hyperalgesia and pain. Current Rheumatology Report. 2008; 10(6):492-9. [DOI:10.1007/s11926-008-0080-z] [PMID] [PMCID]

[20] Rice DA, McNair PJ. Quadriceps arthrogenic muscle inhibition: Neural mechanisms and treatment perspectives. Seminars in Arthritis and Rheumatism. 2010; 40(3):250-66. [DOI:10.1016/j. semarthrit.2009.10.001] [PMID]

[21] Vanderthommen M, Triffaux M, Demoulin C, Crielaard JM, Jean-Loui Croisier JL. Alteration of muscle function after electrical stimulation bout of knee extensors and flexors. Journal of Sports Science and Medicine. 2012; 11(4):592-9. [PMID] [PMCID]

[22] Dehail P, Duclos C, Barat M. Electrical stimulation and muscle strengthening. Annales de Réadaptation et de Médecine Physique. 2008; 51(6):441-51. [DOI:10.1016/j.annrmp.2008.05.001] [PMID]

[23] Dehail P, Duclos C, Barat M. Electrical stimulation and muscle strengthening. Annales de Réadaptation et de Médecine Physique. 2008; 51(6):441-51. [DOI:10.1016/j.annrmp.2008.05.001] [PMID]

[24] Onigbinde AT, Adeloye OO, Akindoyin O, Nesto T. Effects of motor-level transcutaneous electrical nerve stimulation on ipsilateral and un-stimulated contralateral quadriceps femoris. Technology and Health Care. 2014; 22(5):759-66. [DOI:10.3233/ THC-140849] [PMID]

[25] Glaviano NR, Saliba SA. Immediate effect of patterned electrical neuromuscular stimulation on pain and muscle activation in individuals with patellofemoral pain. Journal of Athletic Training. 2016; 51(2):118-28. [DOI:10.4085/1062-6050-51.4.06] [PMID] [PMCID]

[26] Gulick DT, Castel JC, Palermo FX, Draper DO. Effect of patterned electrical neuromuscular stimulation on vertical jump in collegiate athletes. Sports Health. 2011; 3(2):152-7. [DOI:10.1177/1941738110397871] [PMID] [PMCID]

[27] Rosell DR, Custodio RM, Franco-Márquez F, Yáñez-García JM, González Badillo JJ. Traditional vs. sport-specific vertical jump tests: Reliability, validity, and relationship with the legs strength and sprint performance in adult and teen soccer and basketball players. The Journal of Strength and Conditioning Research. 2016; 00(00):1-11. https://www.researchgate.net/publication/303039105

[28] Magdalena Stania , Kajetan J. Stomka, The acute effects of mechanical vs electrical stimuli on countermovement jump. Current Research in Motor Control, 2016; 5:287-93.

[29] Paillard T, Noe F, Bernard N, Dupui P, Hazard C. Effects of two types of neuromuscular electrical stimulation training on vertical jump performance. Journal of Strength and Conditioning Research. 2008; 22(4):1273-8. [DOI:10.1519/JSC.0b013e3181739e9c] [PMID]

[30] Hart JM, Pietrosimone B, Hertel J, Ingersoll CD. Quadriceps activation following knee injuries: A systematic review. Journal of Athletic Training. 2010; 45(1):87-97. [DOI:10.4085/1062-605045.1.87] [PMID] [PMCID] 